\title{
Knockdown of PDX1 enhances the osteogenic differentiation of ADSCs partly via activation of the PI3K/Akt signaling pathway
}

\author{
Fan Liu', Guang-Dong Chen ${ }^{2^{*}}$ and Long-Kun Fan ${ }^{3}$
}

\begin{abstract}
Background: Osteoporosis (OP) is a systemic bone disease manifested as low bone mass, destruction of bone microstructure, increased bone fragility and fracture risk. The purpose of this study was to explore the role and mechanism of PDX1 for osteogenic differentiation of adipose derived stem cells (ADSCs).

Methods: GSE37329 dataset was retrieved from NCBI Gene Expression Omnibus (GEO) database and performed bioinformatic analyses. ADSCs were incubated with normal medium, osteogenic induction medium (OIM) and OIM+si-PDX1. Then, alkaline phosphatase (ALP) staining and Alizarin Red Staining (ARS) were performed to assess the role of PDX1 for osteogenesis of ADSCs. PI3K inhibitor, LY294002 was then added to further explore the mechanism of PDX1 for osteogenic differentiation of ADSCs. Western blot assay was used to assess the osteogenic-related markers. Graphpad software was used to perform statistically analysis.

Results: A total of 285 DEGs were obtained from analysis of the dataset GSE37329, of which 145 were upregulated and 140 were downregulated genes. These differentially expressed genes mainly enriched in cell differentiation and PI3K/Akt signaling pathway. Moreover, PDX1 was decreased in osteogenic induced ADSCs. Knockdown of PDX1 significantly increased osteogenic differentiation capacity and p-PI3K and p-Akt protein levels. Administration with LY294002 could partially reversed the promotion effects of si-PDX1.
\end{abstract}

Conclusion: In conclusion, knockdown of PDX1 promotes osteogenic differentiation of ADSCs through the PI3K/Akt signaling pathway.

Keywords: PDX1, Adipose derived stem cells, Osteogenic differentiation, Bioinformatic analysis

\section{Background}

Osteoporosis is a condition in which bone becomes weak which is characterized as low bone mass and structural deterioration [1]. As a result, bone tissue becomes fragile and shows increased vulnerability to fracture [2]. Worldwide, osteoporosis affects about 200 million people and is often unrecognized until one encounters the fracture due to the silent nature of the disease [3]. Under healthy

\footnotetext{
*Correspondence: chengd2062@sina.com

${ }^{2}$ The Department of Orthopedics, Cangzhou Central Hospital, No. 16 Xinhua West Road, Cangzhou 061000, China

Full list of author information is available at the end of the article
}

conditions, bone is maintained by the constant process of bone remodeling. Normal bone remodeling maintains a balance between bone resorption and formation to maintain bone density [4]. Osteoblasts are generated from the osteogenic differentiation of mesenchymal stem cells (MSCs) [5]. MSCs possess the capacity to self-renew and to differentiate into multiple cell types. It has been known that MSCs are common precursors for osteoblasts [6]. The direction of MSC differentiation depends on specific regulatory factors.

Many factors influenced bone formation by affecting osteogenic differentiation through targeting several pathways in ADSCs. Previously, we found that miR-1249-5p 
regulates the osteogenic differentiation of ADSCs by targeting PDX1 [7]. Role and mechanism of the PDX1 in regulating osteogenic differentiation of ADSCs remains unknown. Pancreatic and duodenal homeobox factor 1 (PDX1) is the most important transcription factor in cell differentiation of multiple stem cells $[8,9]$. However, the role of PDX1 in regulating osteogenic differentiation of ADSCs was unknown.

Many pathways were associated with osteogenic differentiation of ADSCs, including Wnt/ק-catenin [10], PI3K/ Akt [11, 12] and p38-MAPK [13] signaling pathways. PI3K/Akt signaling pathway was one of the most important pathway that involved into cell differentiation [14]. Using a bioinformatics approach, we analyzed the signaling pathway through PDX1 target genes and constructed a protein-protein interaction network.

In this study, we firstly identified the differentially expressed genes between osteogenic induced ADSCs and non-induced ADSCs through GSE37329 datasets. Moreover, following experiments were performed to identify the mechanism of PDX1 in regulating osteogenic differentiation of ADSCs.

\section{Materials and methods Bioinformatic analysis}

GSE37329 was downloaded from Gene Expression Omnibus including 3 ADSCs, 2 ADSCs-derived osteocytes. Background correction and normalization were performed with package 'affy' of R. Missing values were filled with the median method. Differentially expressed genes were identified using the DESeq2 $R$ package and subsequent analysis of gene expression was performed in $R$. Both heatmap and volcano plots were constructed to present the expression profiles of differentially expressed genes using hierarchical clustering, which was performed using the R software. Gene Ontology (GO) and Kyoto Encyclopedia of Genes and Genomes (KEGG) pathway enrichment analysis was performed using the $\mathrm{R}$ package clusterprofiler for genes, to identify over-represented GO terms in three categories (biological processes, molecular function and cellular component), and KEGG pathway. A protein-protein interaction network was acquired using the STRING database with the standard setting. Subnetwork models were selected using the plugin molecular complex detection (MCODE) application in Cytoscape 3.6.1 software (Cytoscape Consortium, California, USA).

\section{ADSCs isolation and culture}

All experiments were confirmed by the Ethical Committees of the Cangzhou Central Hospital. ADSCs were isolated from human adipose tissue obtained from patients who were undergoing total knee arthroplasty at the Department of orthopedic, the XXX Hospital. Adherent cells were cultured in a growth medium [DMEM/F12 (HyClone, USA), 10\% FBS, 1\% Penicillin-Streptomycin Solution (Gibco, USA)] at $37{ }^{\circ} \mathrm{C} / 5 \% \mathrm{CO}_{2}$ and saturated humidity. ADSCs were passaged after reaching 90\% confluence. The antibodies including anti-CD45-FITC, anti-CD45-PE, anti-CD90-FITC and ant-CD105-PE were purchased from BD biosciences (USA). Flow cytometry was used to test these surface markers of mesenchymal stem cells at passage three.

Multi-lineage potential assay was performed to identify osteogenic, adipogenic and chondrogenic phenotype. In brief, ADSCs were cultured into 6-well plates and then transferred to adipogenic medium (Cyagen Biosciences co., LTD, Guangzhou, China), osteogenic medium (Cyagen Biosciences co., LTD, Guangzhou, China) or chondrogenic medium (Cyagen Biosciences co., LTD, Guangzhou, China). At the end of the culture time, ADSCs were stained with ARS, Oil-Red-O staining and Alcian blue staining. Photographs were taken in a light microscope.

\section{Osteogenic differentiation}

The differentiation of cultured ADSCs was induced by incubating osteogenic medium containing BMP-2, FBS (5\%), $\beta$-glycerophosphate $(3 \mathrm{mM})$, and ascorbic acid $(50 \mu \mathrm{g} / \mathrm{mL})$ for 7,14 days. For osteogenic differentiation assays, $2 \times 10^{4}$ cells were seeded in triplicates in a 24-well format plate and cultured in complete medium. Cells maintained in normal growth media were used as the control.

\section{RT-PCR and real-time PCR}

Cells were collected for gene expression profiling related to osteogenic differentiation. To perform qRT-PCR, RNA was isolated using Trizol (Invitrogen). cDNA was produced from $2 \mu \mathrm{g}$ RNA with reverse transcriptase as described by the M-MLV manual (New England Biolabs). For RT-PCR, PDX1 primers were used. The relative quantity of PDX1 was normalized to GAPDH. The expression of PDX1 was detected using qPCR with SYBR Green Mix Kits (Applied Biosystems). All results were quantitated using the $2^{-\Delta \Delta C t}$ relative quantification method.

\section{Western blot analysis}

Total proteins were extracted with lysis buffer containing PMSF and RIPA (PMSF: RIPA =1: 99) and then qualified with the Bicinchoninic Acid (BCA) assay kit (Beyotime, Beijing, China). Thereafter, protein extracts $(20-40 \mu \mathrm{g})$ were separated on a $10 \%$ sodium dodecyl sulfate polyacrylamide gel electrophoresis, transferred onto a nitrocellulose membrane and then blocked with $5 \%$ milk for $1 \mathrm{~h}$. Next, the membrane was probed with primary antibodies overnight at $4{ }^{\circ} \mathrm{C}$, followed by the corresponding 

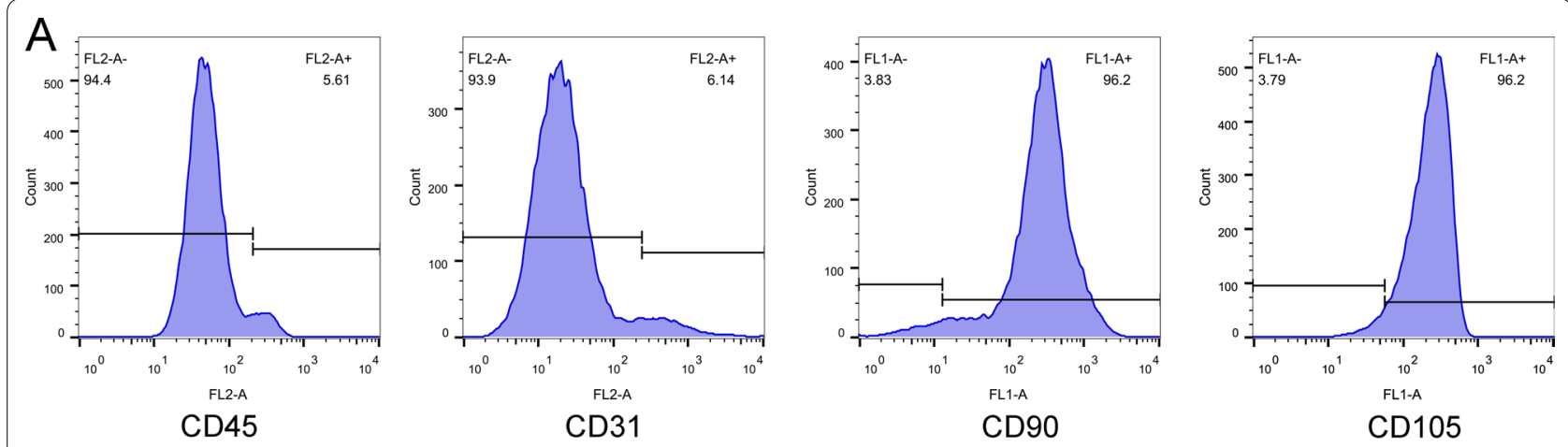

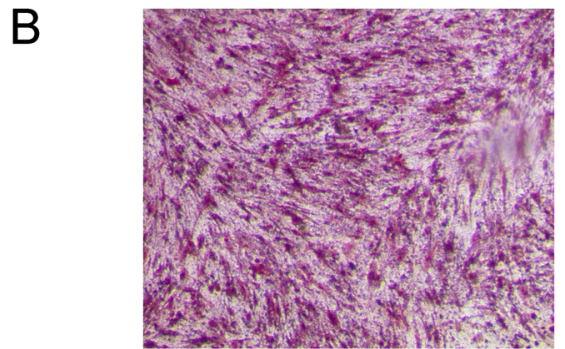

Alizarin Red

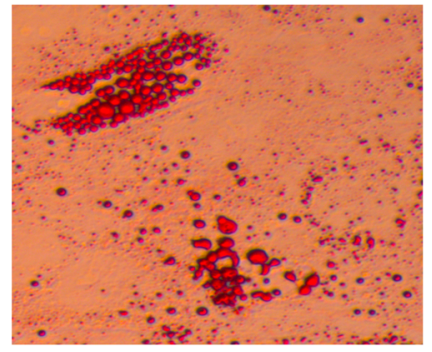

Oil Red O

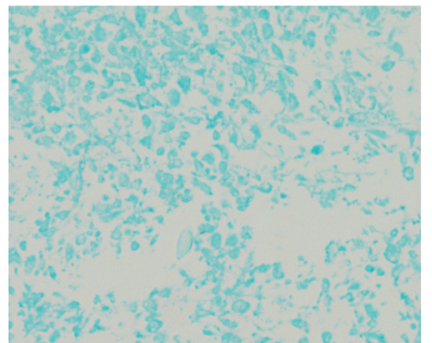

Alcian Blue

Fig. 1 Identification of human ADSCs. A Flow cytometric analysis of ADSC surface markers (CD45, CD31, CD90 and CD105). B Osteogenic, adipogenic and chondrogenic differentiation of ADSCs

secondary antibody for $1 \mathrm{~h}$ at $37^{\circ} \mathrm{C}$. The blots were visualized using enhanced chemiluminescence reagents (BeyoECL Plus; Beyotime Institute of Biotechnology). All antibodies were bought from Abcam (Cambridge, MA, USA): OSX (1:5000), OCN (1:5000), ALP (1:5000), RUNX2 (1:2000), and GAPDH (1:5000).

\section{PDX1 knockdown}

The siRNA target sequence was designed against human PDX1. For transient transfection, PDX1 sequence was retrieved from GenBank, and two siRNA sequences were designed, namely, PDX-1 siRNA-1 (5'-AGCCAAGAG AGGCATAGA-3), PDX-1 siRNA-2 (5'-AGCTACCCG GAAGACAG-3). Transfection efficiency was controlled by evaluating PDX1 level using RT-PCR and Western Blot analysis after $48 \mathrm{~h}$.

For the knockdown experiment, 50\% confluent ADSCs were transfected using Scrambled siRNA and designed PDX1-specific siRNAs using Lipofectamine RNAiMAX reagent (Invitrogen, USA). The efficiency of knockdown of PDX1 was confirmed 2 days after siRNA transfection. To further assess the PDX1 for the influence of PI3K/Akt signaling pathway, LY294002 at a concentration of $10 \mu \mathrm{M}$ and $50 \mu \mathrm{M}$ for $12 \mathrm{~h}$ before exposure to OIM according to previous literature [15]. The inhibitor efficacy was further identified by western blot to assess the protein level of
p-Akt. PDX1 overexpression plasmid (Flag- PDX1) was purchased from Origene Technologies (Origene).

\section{Alkaline phosphatase (ALP) activity}

After treatment, each plate was fixed by $4 \%$ paraformaldehyde for 10 min and then washed with PBS for three times. Cells were stained with 5-bromo-4-chloro-3-indolylphosphate (BCIP) and 4-nitro blue tetrazolium chloride (NBT) for $30 \mathrm{~min}$. Then the reactions were stopped with distilled water. Observation and photographing were performed by a light microscope (Olympus, Milan, Italy). ALP activity was measured and the results were normalized to levels of total protein.

\section{Alizarin red S staining}

Matrix mineralization was assessed through staining with Alizarin Red S (Solarbio, Beijing, China). After stimulation, the cells were washed, followed by fixation with ethanol (70\%) for $45 \mathrm{~min}$. Cells were then stained with the dye Alizarin Red S. Fluorescence signals were visualized using a fluorescence microscope.

\section{Statistical analysis}

All experiments were performed in triplicate. The data were displayed as the mean \pm standard deviation (SD) and processed by GraphPad Prism 7 software. Student's $t$ test or one-way analysis of variance (ANOVA) was 


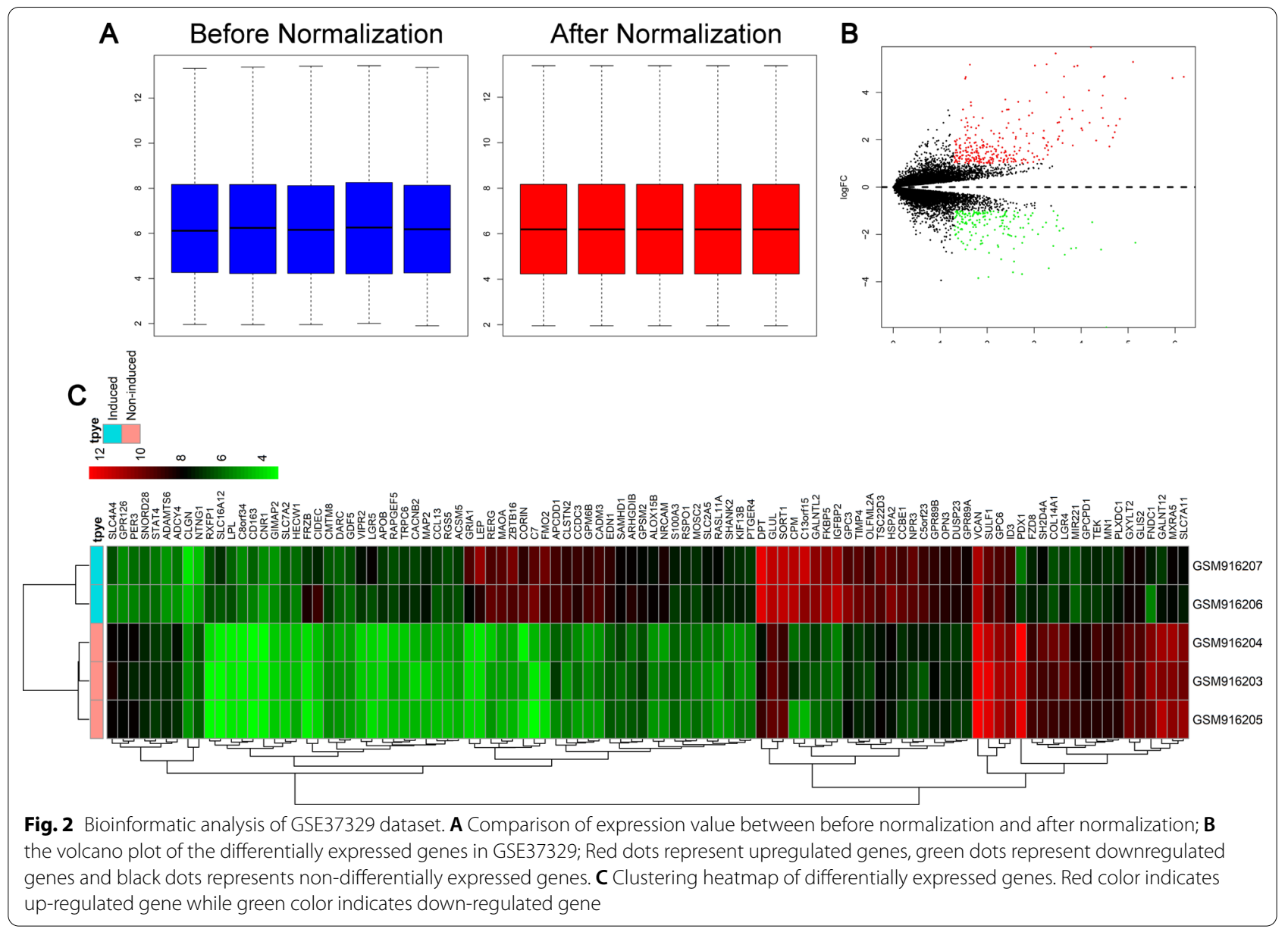

utilized to analyze significant differences. The $P<0.05$ indicated statistically significant.

\section{Results}

\section{Bioinformatic analysis results}

Box plots before and after normalization of the raw data is shown in Fig. 1A. The median values of each sample were extremely similar, indicating that the data should be further analyzed. A total of 285 DEGs were obtained from analysis of the dataset GSE37329, of which 145 were upregulated and 140 were downregulated genes. The volcano plot and heatmap of differentially expressed genes is presented in Fig. 2B and $\mathrm{C}$ respectively. we found the gene list to be enriched in biological processes including 204 genes mainly involved in response to lipopolysaccharide, extracellular matrix organization, cell-cell signaling, positive regulation of cytosolic calcium ion concentration, signal transduction, negative regulation of cell proliferation, negative regulation of transcription from RNA polymerase II promoter, fat cell differentiation, positive regulation of macrophage derived foam cell differentiation and negative regulation of smooth muscle cell proliferation.

Cellular component mainly enriched in extracellular space, extracellular region, proteinaceous extracellular matrix, plasma membrane, integral component of plasma membrane, extracellular exosome, cell surface, organelle membrane, platelet alpha granule lumen, anchored component of membrane. Molecular function terms mainly enriched in cytokine activity, heparin binding, chemorepellent activity, receptor binding, growth factor activity, transforming growth factor beta receptor binding, PDZ domain binding, Wnt-protein binding, transcription corepressor activity and N,N-dimethylaniline monooxygenase activity. KEGG pathways mainly enriched in the cGMP-PKG signaling pathway, neuroactive ligand-receptor interaction, signaling pathways regulating pluripotency of stem cells, PI3K/Akt signaling pathway, vascular smooth muscle contraction, TGF-beta signaling pathway, NF-kappa B signaling pathway, inflammatory mediator regulation of TRP channels, circadian entrainment and Rap1 signaling pathway. 


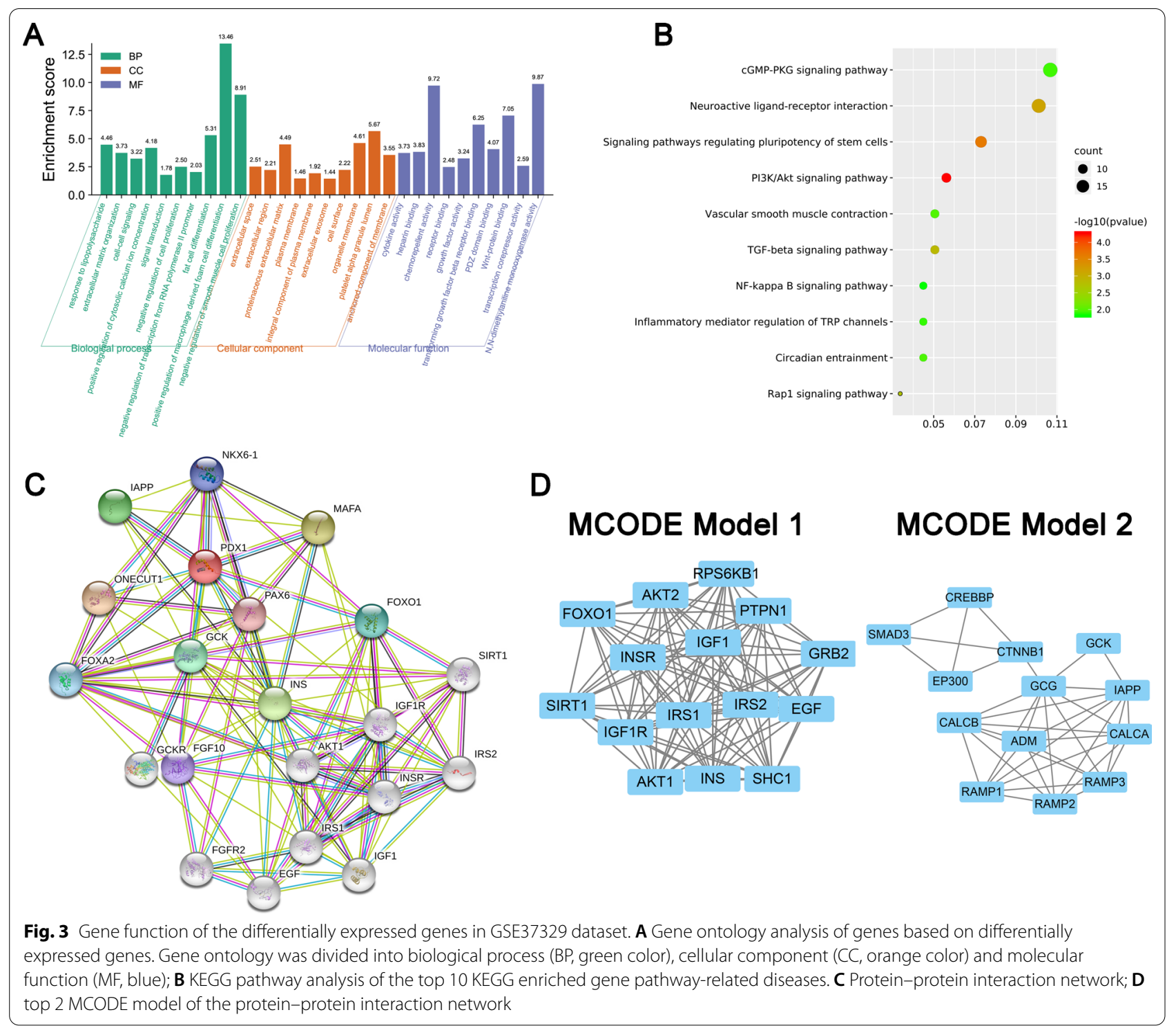

Figure $1 \mathrm{C}$ represents the protein-protein interaction network of differentially expressed genes having 18 nodes and 86 edges. As illustrated in Fig. 1D, the top two functional clusters of modules were selected (module 1, MCODE score =18.357; module 2, MCODE score $=10.333)$.

\section{Identification of ADSCs}

As illustrated in Fig. 2A, ADSCs expressed stem cellassociated markers CD90 and CD105 and negative expressed with CD45 and CD31. Under proper stimulation, ADSCs should be able to differentiate into osteogenic, adipogenic and chondrogenic progenies (Fig. 2B). Compared with control group, ADSCs that underwent osteogenic differentiation possess higher expression of PDX1 with statistically significant ( $P<0.05$, Fig. 3$)$.

\section{Knockdown of PDX1 increase the osteoblastic differentiation capacity of ADSCs}

After transfection with PDX1 siRNA (PDX1 siRNA-1 or PDX1 siRNA-2), the PDX1 mRNA and protein expression was significantly downregulated (Additional file 1: Fig. S1A and B). ADSCs were divided into three groups: control, OIM and OIM+si-PDX1 groups. Then, ALP staining and ARS were performed to assess the early and late osteogenic ability respectively. Compared with control group, ADSCs cultured with OIM significantly increase the ALP activity and calcium deposition at 7 and 21 days, respectively. However, when administration 


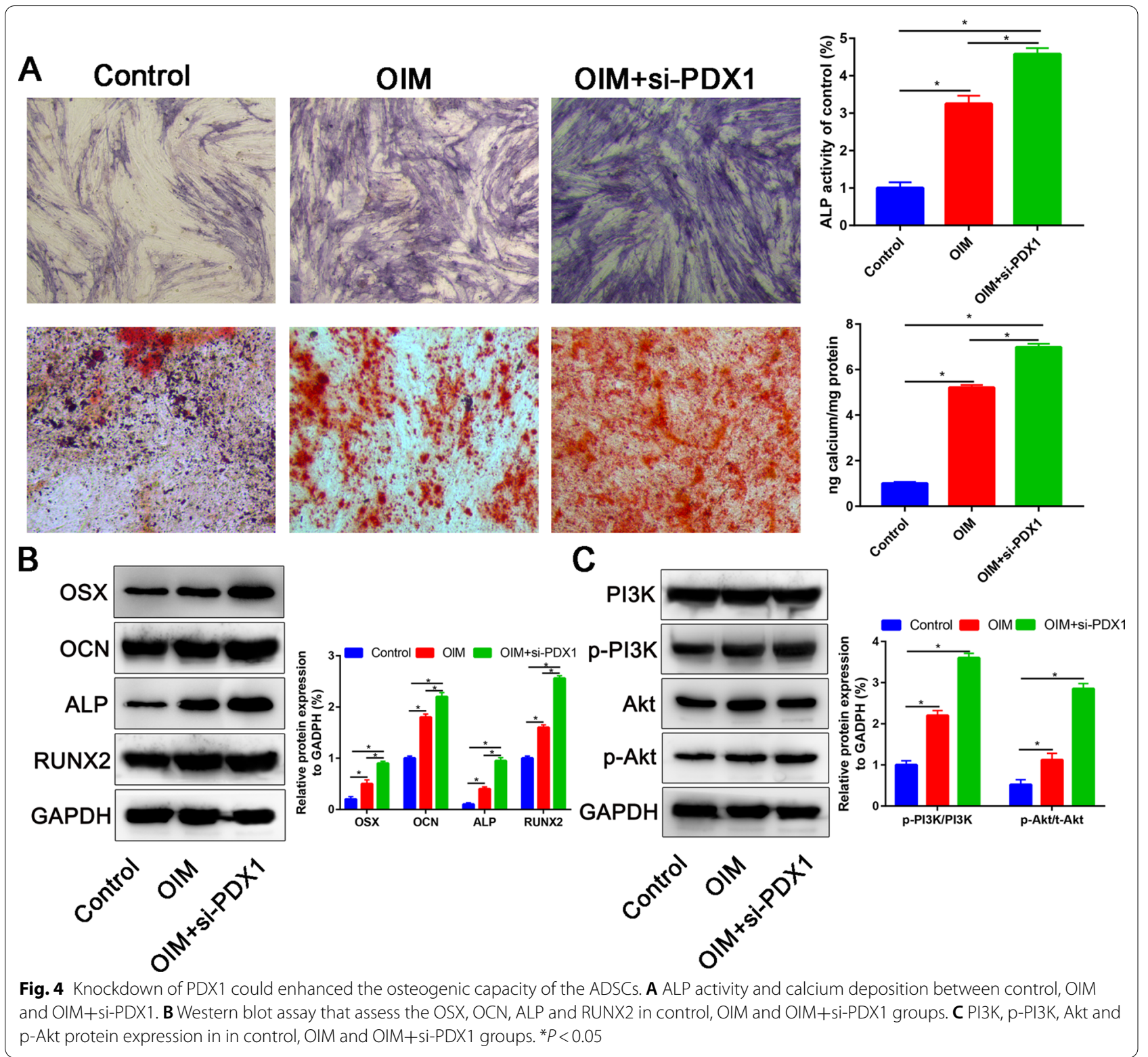

with si-PDX1, the ALP activity and calcium deposition was partially attenuated than OIM alone group (Fig. 4A). Then, we performed western blot assay to assess the osteogenic-related markers (OSX, OCN, ALP and RUNX2) in each group. ADSCs cultured with OIM significantly increase the OSX, OCN, ALP and RUNX2 expression, while co-cultured with si-PDX1 partially attenuated these osteogenic-related markers expression (Fig. 4B). To further explore the mechanism of PDX1 for osteogenic differentiation of ADSCs, we assessed the PI3K/Akt pathway related markers change. We found that after knockdown of PDX1 could significantly activate the PI3K/Akt signaling pathway. ADSCs were transfected with PDX1
siRNA or control siRNA (control) for different times. Then, we assessed the p-PI3K and p-Akt expression in different treatment groups. In terms of increasing incubation duration, $\mathrm{p}$-PI3K and $\mathrm{p}$-Akt showed a progressive increasing trend (Additional file 2: Fig. S2).

\section{Overexpression PDX1 decrease the osteoblastic differentiation capacity of ADSCs}

After transfection with PDX1 plasmid, ALP activity and calcium deposition was partially decreased (Fig. 5A, $P<0.05)$. Consistent with ALP activity and ARS staining, the OSX, OCN, ALP and RUNX2 were significantly 


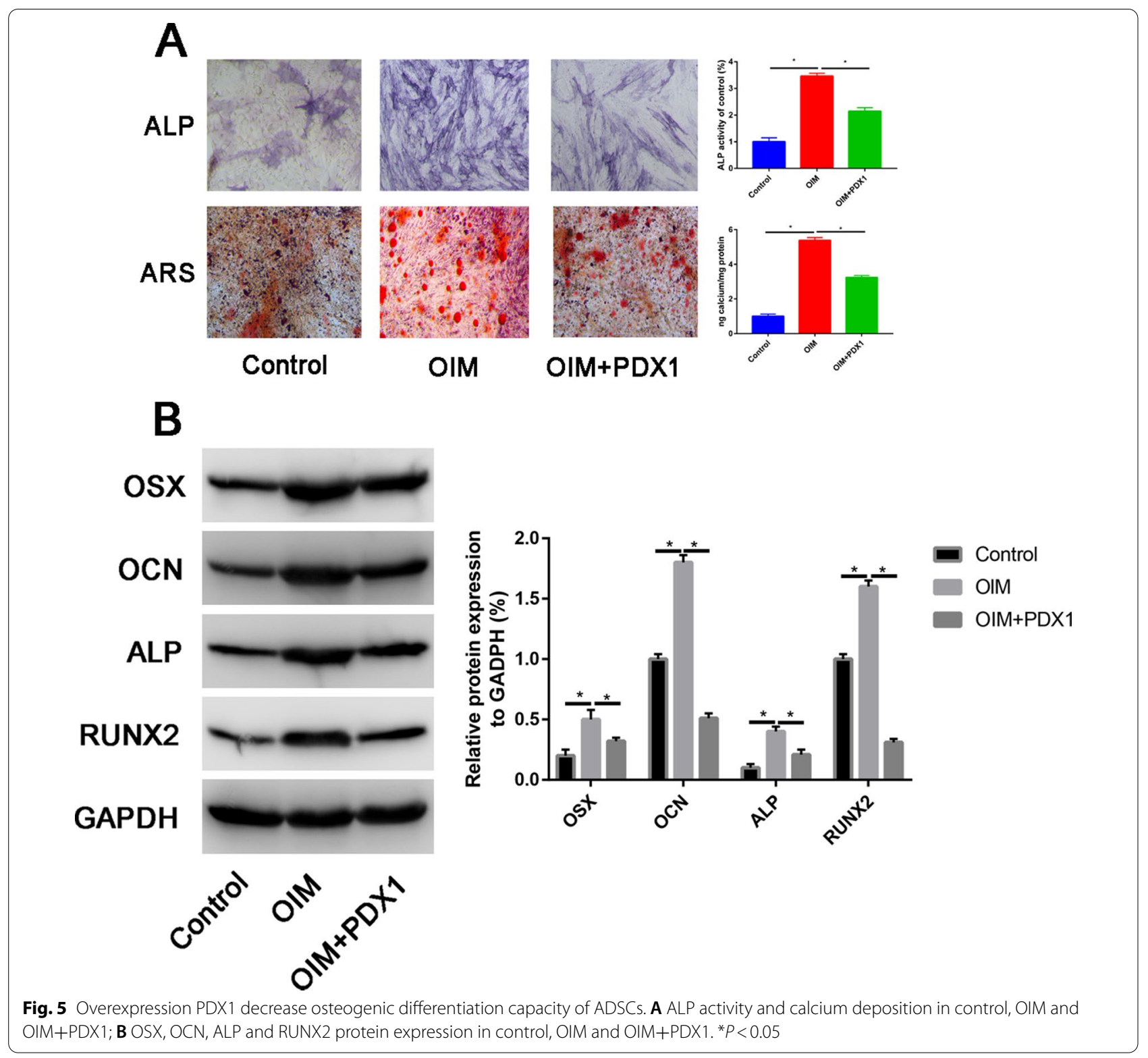

downregulated in PDX1 group than OIM group (Fig. 5B, $P<0.05)$.

\section{LY294002 could partially reversed the promotion effects of si-PDX 1}

To further explore the mechanism of PDX1 for osteogenic differentiation of ADSCs. We administrated PI3K inhibitor, LY294002 to assess the osteogenic differentiation ability of ADSCs. Western blot showing that p-AKT expression decreased with LY294002 treatment (Additional file 3: Fig. S3). There was no significant difference between $10 \mu \mathrm{M}$ and $50 \mu \mathrm{M}$ LY294002 $(P>0.05)$.
ALP and ARS staining found that administration LY294002 could partially reverse the ALP activity and calcium deposition after OIM induction (Fig. 6A). Further western blot assay found that LY294002 could partially downregulated the osteogenic related markers expression (OSX, OCN, ALP and RUNX2, Fig. 6B).

\section{Discussion}

In this study, we firstly assessed the role of PDX1 for the osteogenic differentiation of ADSCs. We found that PDX1 was differentially expressed between OIM and normal medium. Further studies found that knockdown of PDX1 significantly increase the osteogenic capacity 


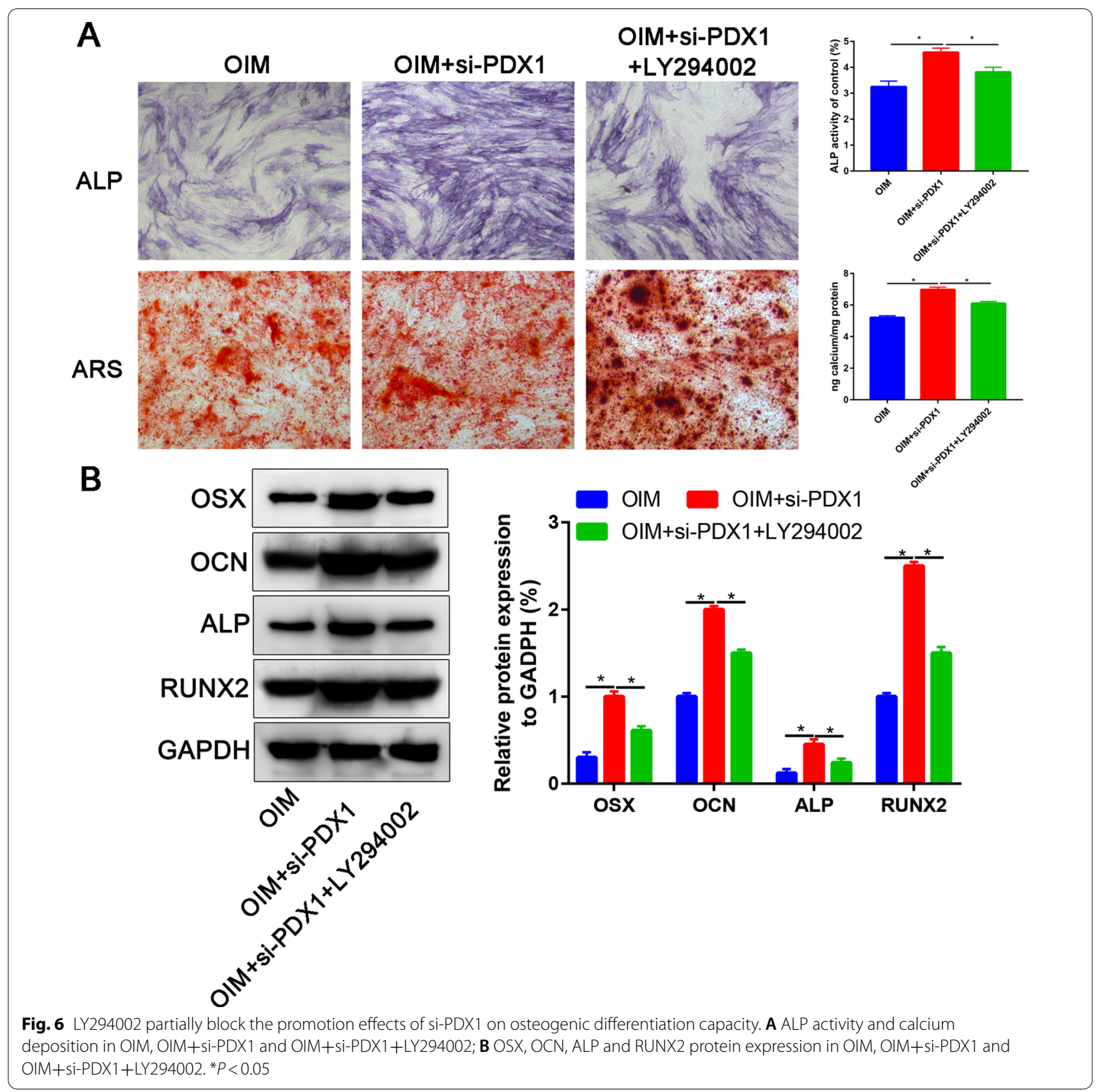

of ADSCs. Moreover, administrated with PI3K inhibitor could partially reversed the promotion effects of si-PDX1.

We firstly analyses the GSE37329 datasets, which containing 2 osteogenic induced ADSCs and 3 noninduced ADSCs. Further in-depth studies revealed that PDX1 may interact with the PI3K/Akt signaling pathway to promote the osteogenic differentiation of ADSCs. ADSCs are multipotent stem cells, which can differentiate into osteoblasts, odontoblasts and adipocytes in specific medium. ADSCs have become promising seed cells for bone tissue engineering due to their easy access and availability in large quantities [16]. It has been reported that ADSCs tend to differentiate into adipocytes rather than osteoblasts [17].

For ADSCs-based therapy to be successful in in vivo treatment of bone diseases, it is crucial to transplant ADSCs with a suitable substance that facilitates their osteogenic differentiation in vivo $[18,19]$. Firstly, knockdown of PDX1 could significantly increase the osteogenic capacity, which suggested that PDX1 is emerging as a promising therapeutic target in bone disease therapy. PDX1 is a regulator of pancreas development and $\beta$ cell 
differentiation [20]. Further study found that the increase in PDX1 levels is crucial for the development and differentiation of pancreatic $\beta$ cells [21]. Moreover, PDX1 is a critical transcription regulators for beta cell development and regeneration [22].

Several signaling pathways are involved in ADSCs osteogenic differentiation, including the ERK1/2 [23], Wnt [6], phosphatidylinositide-3 kinase (PI3K)/Akt [24], and BMP-Smad pathways [25]. However, the mechanism of PDX1 for osteogenic differentiation of ADSCs is largely unknown. We found that PDX1 knockdown significantly activate the PI3K/Akt signaling pathway. Previously, Jara et al. [26] found that PDX1 contributes to $\beta$-cell mass expansion and proliferation induced by Akt/PKB pathway. These results suggested that PDX1 directly or indirectly affect the PI3K/Akt signaling pathway. In this study, we found that knockdown of PDX1 significantly activate the PI3K/Akt signaling pathway and finally promote the osteogenic differentiation of ADSCs. The PI3K/AKT pathway plays a crucial role in osteogenic differentiation of ADSCs and acts as a chief regulator in bone cells' proliferation and metabolism. Our data revealed that si-PDX1 through PI3K/AKT signaling pathways trigger osteogenic differentiation of ADSCs.

As revealed in the present study, the knockdown of PDX1 increased the levels of p-Akt and p-PI3K via the PI3K/AKT pathway in ADSCs, which was decreased by treatment with the PI3K inhibitor LY294002. Previous studies have revealed that enhanced osteoinductivity was induced through activating the PI3K/Akt signaling pathway [27-29]. Protein-protein interaction revealed that PDX1 could directly regulating PI3K/Akt signaling pathway. Western blot assay and PI3K inhibitor experiments further confirmed that PDX1 could affect the PI3K/Akt signaling pathway and finally influence the osteogenic differentiation of ADSCs.

The main limitation of the present study was the lack of in vivo experiments. Moreover, the downstream signaling pathways were not explained in detail. Multiple pathways involved into the osteogenic differentiation of ADSCs and the correlation between these pathways need to be identified.

\section{Conclusion}

In conclusion, this study firstly demonstrated that knockdown of PDX1 could enhance the osteogenesis of ADSCs in vitro through PI3K/Akt signaling pathway. PDX1 was identified as novel target of bone-related disease. Our study provided a new insight into the treatment of OP.

\section{Abbreviations}

OP: Osteoporosis; ADSCs: Adipose derived stem cells; GEO: Gene expression omnibus; OIM: Osteogenic induction medium; ALP: Alkaline phosphatase; ARS: Alizarin red staining; MSCs: Mesenchymal stem cells; PDX1: Pancreatic and duodenal homeobox factor 1; GO: Gene ontology; KEGG: Kyoto encyclopedia of genes and genomes; MCODE: Molecular complex detection; BCA: Bicinchoninic acid; BCIP: 5-Bromo-4-chloro-3-indolylphosphate; NBT: 4-Nitro blue tetrazolium chloride; SD: Standard deviation; ANOVA: One-way analysis of variance.

\section{Supplementary Information}

The online version contains supplementary material available at https://doi. org/10.1186/s13018-021-02825-4.

Additional file 1. A, Relative PDX1 expression in siRNA control, PDX-1 siRNA-1 and PDX-1 siRNA-2 groups.

Additional file 2. Relative p-PI3K, PI3K, p-Akt and Akt expression in control and PDX-1 treatment group with different treatment.

Additional file 3. Relative Akt and p-Akt expression in control and LY294002 (10 $\mu \mathrm{M}$ and $50 \mu \mathrm{M})$ groups.

Acknowledgements

Not applicable.

Authors' contributions

FL and GDC design the study and conducted the experiments. FL and LKF perform the statistically analyses. FL and LKF write the draft. FL and GDC edited and confirmed the final manuscript. All authors read and approved the final manuscript.

Funding

There is no funding for this article.

Availability of data and materials

We state that the data will not be shared since all the raw data are present in the figures included in the article.

\section{Declarations}

Ethics approval and consent to participate

Not applicable.

Consent for publication

Not applicable.

\section{Competing interests}

The authors declare that they have no competing interests.

\section{Author details}

${ }^{1}$ The First Department of Anesthesiology, Cangzhou Central Hospital, Cangzhou 061000, China. ${ }^{2}$ The Department of Orthopedics, Cangzhou Central Hospital, No. 16 Xinhua West Road, Cangzhou 061000, China. ${ }^{3}$ The Department of Medical Plastic Surgery, Cangzhou Central Hospital, Cangzhou 061000, China.

Received: 9 August 2021 Accepted: 1 November 2021

Published online: 19 February 2022

\section{References}

1. Fusaro M, Cianciolo G, Brandi ML, et al. Vitamin K and osteoporosis. Nutrients. 2020;12:E3625. https://doi.org/10.3390/nu12123625.

2. Liu Y, Wang H, Zhou XZ, et al. Pentraxin 3 promotes the osteoblastic differentiation of MC3T3-E1 cells through the PI3K/Akt signaling pathway. Biosci Rep. 2020. https://doi.org/10.1042/bsr20201165 
3. LiW, Chen Z, Cai C, et al. MicroRNA-505 is involved in the regulation of osteogenic differentiation of MC3T3-E1 cells partially by targeting RUNX2. J Orthop Surg Res. 2020;15:143. https://doi.org/10.1186/ s13018-020-01645-2.

4. Zhao Z, Ma X, Ma J, et al. Naringin enhances endothelial progenitor cell (EPC) proliferation and tube formation capacity through the CXCL12/ CXCR4/PI3K/Akt signaling pathway. Chem Biol Interact. 2018;286:45-51. https://doi.org/10.1016/j.cbi.2018.03.002.

5. Skubis-Sikora A, Sikora B. Osteogenesis of adipose-derived stem cells from patients with glucose metabolism disorders. Mol Med. 2020;26:67. https://doi.org/10.1186/s10020-020-00192-0.

6. Li Y, Fu H, Wang $\mathrm{H}$, et al. GLP-1 promotes osteogenic differentiation of

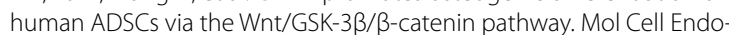
crinol. 2020;515: 110921. https://doi.org/10.1016/.mce.2020.110921.

7. Yang XM, Song YQ, Li L, et al. miR-1249-5p regulates the osteogenic differentiation of ADSCs by targeting PDX1. J Orthop Surg Res. 2021;16:10. https://doi.org/10.1186/s13018-020-02147-x

8. He D, Wang J, Gao Y, et al. Differentiation of PDX1 gene-modified human umbilical cord mesenchymal stem cells into insulin-producing cells in vitro. Int J Mol Med. 2011;28:1019-24. https://doi.org/10.3892/ijmm. 2011.774

9. Court $\mathrm{H}$, Ahearn IM, Amoyel M. Regulation of NOTCH signaling by RAB7 and RAB8 requires carboxyl methylation by ICMT. J Cell Biol. 2017;216:4165-82. https://doi.org/10.1083/jcb.201701053.

10. Wang Y, Zhang X, Shao J, et al. Adiponectin regulates BMSC osteogenic differentiation and osteogenesis through the Wnt/ $\beta$-catenin pathway. Sci Rep. 2017;7:3652. https://doi.org/10.1038/s41598-017-03899-z.

11. Ye C, Zhang W, Hang K, et al. Extracellular IL-37 promotes osteogenic differentiation of human bone marrow mesenchymal stem cells via activation of the PI3K/AKT signaling pathway. Cell Death Dis. 2019;10:753. https://doi.org/10.1038/s41419-019-1904-7.

12. Wu R, Ruan J, Sun Y, et al. Long non-coding RNA HIF1A-AS2 facilitates adipose-derived stem cells (ASCs) osteogenic differentiation through miR-665/IL6 axis via PI3K/Akt signaling pathway. Stem Cell Res Ther. 2018;9:348. https://doi.org/10.1186/s13287-018-1082-z.

13. Liu Q, Zhuang Y, Ouyang N, et al. Cytochalasin D promotes osteogenic differentiation of MC3T3-E1 cells via P38-MAPK signaling pathway. Curr Mol Med. 2019;20:79-88. https://doi.org/10.2174/156652401966619 1007104816.

14. Dong K, Hao P, Zhou W, et al. Concentrate growth factors regulate osteogenic dysfunction of MC3T3-E1 cells induced by high glucose through PI3K/Akt signaling pathway. Implant Dent. 2019;28:478-83. https://doi. org/10.1097/id.0000000000000921.

15. Chen QJ, Chen L, Wu SK, et al. rhPDGF-BB combined with ADSCs in the treatment of Achilles tendinitis via miR-363/PI3 K/Akt pathway. Mol Cell Biochem. 2018;438:175-82. https://doi.org/10.1007/s11010-017-3124-8,

16. Li Q, Yang G, Li J, et al. Stem cell therapies for periodontal tissue regeneration: a network meta-analysis of preclinical studies. Stem Cell Res Ther. 2020;11:427. https://doi.org/10.1186/s13287-020-01938-7.

17. Yang S, Guo S. Promoting osteogenic differentiation of human adiposederived stem cells by altering the expression of exosomal miRNA. Stem Cells Int. 2019;2019:1351860. https://doi.org/10.1155/2019/1351860.

18. Park JS, Piao J, Park G, et al. Osteoporotic conditions influence the activity of adipose-derived stem cells. Tissue Eng Regen Med. 2020;17:875-85. https://doi.org/10.1007/s13770-020-00289-x.

19. Zhang D, Ni N, Wang Y, et al. CircRNA-vgll3 promotes osteogenic differentiation of adipose-derived mesenchymal stem cells via modulating miRNA-dependent integrin a5 expression. Cell Death Differ. 2020. https:// doi.org/10.1038/s41418-020-0600-6.

20. Walter Costa MB, Höner Zu Siederdissen C. SSS-test: a novel test for detecting positive selection on RNA secondary structure. BMC Bioinform. 2019:20:151. https://doi.org/10.1186/s12859-019-2711-y.

21. Yu H, Liu J, Li X, et al. Identification of a novel mutation site in maturityonset diabetes of the young in a Chinese family by whole-exome sequencing. Mol Med Rep. 2019;20:2373-80. https://doi.org/10.3892/ mmr.2019.10464.

22. Zhu Y, Liu Q, Zhou Z, et al. PDX1, Neurogenin-3, and MAFA: critical transcription regulators for beta cell development and regeneration. Stem Cell Res Ther. 2017:8:240. https://doi.org/10.1186/s13287-017-0694-z.

23. Fathi E, Farahzadi R. Enhancement of osteogenic differentiation of rat adipose tissue-derived mesenchymal stem cells by zinc sulphate under electromagnetic field via the PKA, ERK1/2 and Wnt/ $\beta$-catenin signaling pathways. PLoS ONE. 2017;12: e0173877. https://doi.org/10.1371/journal. pone. 0173877

24. Tang L, Wu M, Lu S, et al. Fgf9 negatively regulates bone mass by inhibiting osteogenesis and promoting osteoclastogenesis via MAPK and PI3K AKT signaling. J Bone Miner Res. 2020. https://doi.org/10.1002/jbmr.4230.

25. Tang J, Qing MF, Li M, et al. Dexamethasone inhibits BMP7-induced osteogenic differentiation in rat dental follicle cells via the PI3K/AKT/GSK3ß/ß-catenin pathway. Int J Med Sci. 2020;17:2663-72. https://doi.org/10. 7150/ijms.44231.

26. Jara MA, Werneck-De-Castro JP. Pancreatic and duodenal homeobox-1 (PDX 1) contributes to $\beta$-cell mass expansion and proliferation induced by Akt/PKB pathway. Islets. 2020;12:32-40. https://doi.org/10.1080/19382 014.2020.1762471.

27. Chen $Y$, Hu Y, Yang L, et al. Run $\times 2$ alleviates high glucose-suppressed osteogenic differentiation via PI3K/AKT/GSK3 $\beta / \beta$-catenin pathway. Cell Biol Int. 2017:41:822-32. https://doi.org/10.1002/cbin.10779.

28. Zhu X, Zhou L, Liu Z, et al. Telomerase enhances osteogenic ifferentiation of sheep bone marrow mesenchymal stem cells (BMSCs) by up-regulating PI3K/Akt pathway in vitro. Pol J Vet Sci. 2020;23:359-72. https://doi. org/10.24425/pjvs.2020.133653.

29. Zhang $S$, Chen $X$, Hu Y, et al. All-trans retinoic acid modulates Wnt3Ainduced osteogenic differentiation of mesenchymal stem cells via activating the PI3K/AKT/GSK3ß signalling pathway. Mol Cell Endocrinol. 2016;422:243-53. https://doi.org/10.1016/j.mce.2015.12.018.

\section{Publisher's Note}

Springer Nature remains neutral with regard to jurisdictional claims in published maps and institutional affiliations.

Ready to submit your research? Choose BMC and benefit from

- fast, convenient online submission

- thorough peer review by experienced researchers in your field

- rapid publication on acceptance

- support for research data, including large and complex data types

- gold Open Access which fosters wider collaboration and increased citations

- maximum visibility for your research: over $100 \mathrm{M}$ website views per year

At BMC, research is always in progress.

Learn more biomedcentral.com/submissions 